\title{
SOME RESULTS ON SYMMETRIC DUALITY OF MATHEMATICAL FRACTIONAL PROGRAMMING WITH GENERALIZED F-CONVEXITY COMPLEX SPACES
}

\author{
DEO BRAT OJHA
}

\begin{abstract}
In this present article we have given some mathematical fractional programming problems with their symmetric duals and have derived weak and strong duality results with respect to such programs. Moreover, we have also used most general type of convexity assumptions involved with the functions which are related to the programming problems. It is to be pointed out that the objective functions in such programs contain terms like support functions which in turn are able to give results on particular classes of programs involving quadratic terms. Our results in particular give as of special cases some eariler results symmetric duals given in the current literature. All discussion goes to complex spaces.
\end{abstract}

\section{Introduction}

In a very recent work [6] have given some results on symmetric duals for mathematical programming problems where $F$-convexity concepts have been incorporated. Some kind of weak duality results and strong duality theorem have been derived under generalized $F$-convexity conditions. It may be observed that in ref.[6], the authors have shown that some previous results can be obtained as special cases of their results, e.g. if there is a quadratic term like $\left(X^{T} A X\right)^{1 / 2}$ in the objective then the specializing the program of the above references, one can get results for duality theorems with objective having quadratic terms.

Again in very recent article [3], authors moves real spaces to complex spaces for discussing symmetric duality of mathematical programming with $F$-convexity, which is the enhanced discussion to improve this field significantly.

In the present article, we are giving symmetric dual of a class of mathematical fractional programming problems under $F$-convexity assumptions in complex space in two variables which have wider applications and as an outcome weak and strong duality theorems have been derived and elaborated.

Received February 10, 2004; revised September 10, 2004.

2000 Mathematics Subject Classification. 90C30.

Key words and phrases. Mathematical programming, symmetric duality, F-convexity. 


\section{Notation and Preliminaries}

Let $C^{n}$ denote an $n$-dimensional complex space. For $x \in C^{n}$, let the real vectors $\operatorname{Re}(x)$ and $\operatorname{Im}(x)$ denote the real and imaginary parts, respectively, and let $\bar{x}=\operatorname{Re}(x)-i \operatorname{Im}(x)$ be the conjugate of $x$. Given a matrix $A=\left[a_{i j}\right] \in C^{m \times n}$, where $C^{m \times n}$ is the collection of $m \times n$ complex matrices, let $\bar{A}=\left\lfloor\bar{a}_{i j}\right\rfloor \in C^{m \times n}$ denote its conjugate matrix, let $A^{H}=\left[\bar{a}_{j i}\right]$ denote its conjugate transpose. The inner product of $x, y \in C^{n}$ is $(x, y)=y^{H} x$. Let $R_{+}$ denote the halfline $[0, \infty[$.

For a complex function $f: C^{n} \times C^{n} \times C^{m} \times C^{m} \rightarrow C$ analytic with respect to $\zeta=\left(w^{1}, w^{2}\right), z \in C^{n}$, define gradients by

$$
\nabla_{z} f(v, \bar{v}, \zeta)=\left[\frac{\partial f}{\partial w^{1}}(v, \bar{v}, \zeta)\right], \quad \nabla_{\bar{z}} f(v, \bar{v}, \zeta)=\left[\frac{\partial f}{\partial w^{2}}(v, \bar{v}, \zeta)\right]
$$

In order to define generalized $F$-convexity, we introduced the functional $F$ as follows.

Let $F: C^{n} \times C^{n} \times C^{n} \rightarrow R$ be sub linear on the third variable. Then we can define generalized $F$-convexity for analytic functions.

Definition 2.1. The real part Re $f$ of an analytic function $f: C^{n} \times C^{n} \times C^{m} \times C^{m} \rightarrow$ $C$ is said to be $F$-convex at $(u, \bar{u})$ with respect to $R_{+}$if for any $x \in C^{n}$ for fixed $(y, \bar{y}) \in C^{2 m}, \operatorname{Re}[f(x, \bar{x}, y, \bar{y})-f(u, \bar{u}, y, \bar{y})] \geq F\left(x, u, \bar{\nabla}_{x} f(u, \bar{u}, y, \bar{y})+\nabla_{\bar{x}} f(u, \bar{u}, y, \bar{y})\right)$ for some arbitrary sublinear functional $F$.

Remark. The above definition is given in [3].

Definition 2.2. The real part Re $f$ of an analytic function $f: C^{n} \times C^{n} \times C^{m} \times C^{m} \rightarrow$ $C$ is said to be $F$-concave at $(y, \bar{y})$ with respect to $R_{+}$if for any $x \in C^{n}$ for fixed $(x, \bar{x}) \in C^{n} \times C^{n}$, if

$$
\operatorname{Re}[f(x, \bar{x}, y, \bar{y})-f(x, \bar{x}, u, \bar{u})] \geq F\left(y, u,-\overline{\nabla_{y} f(x, \bar{x}, y, \bar{y})}-\nabla_{\bar{y}} f(x, \bar{x}, y, \bar{y})\right)
$$

for some arbitrary sublinear functional $F$.

Definition 2.3. The real part Re $f$ of an analytic function $f: C^{n} \times C^{n} \times C^{m} \times C^{m} \rightarrow$ $C$ is said to be $F$-pseudoconvex at $(u, \bar{u})$ with respect to $R_{+}$if for any $x \in C^{n}$ for fixed $(y, \bar{y}) \in C^{m} \times C^{m}$, if

$$
F\left(x, u, \overline{\nabla_{x} f(u, \bar{u}, y, \bar{y})}+\nabla_{\bar{x}} f(u, \bar{u}, y, \bar{y})\right) \geq 0 \Rightarrow \operatorname{Re}[f(x, \bar{x}, y, \bar{y})-f(u, \bar{u}, y, \bar{y})] \geq 0
$$

for all $x \in C^{n}$ for some arbitrary sublinear functional $F$.

Definition 2.4. The real part Re $f$ of an analytic function $f: C^{n} \times C^{n} \times C^{m} \times C^{m} \rightarrow$ $C$ is said to be $F$-pseudoconcave at $(u, \bar{u})$ with respect to $R_{+}$if for any $x \in C^{n}$ for fixed $(x, \bar{x}) \in C^{n} \times C^{n}$, if

$$
F\left(y, u,-\overline{\nabla_{y} f(x, \bar{x}, u, \bar{u})}-\nabla_{\bar{y}} f(x, \bar{x}, u, \bar{u})\right) \geq 0 \Rightarrow \operatorname{Re}[f(x, \bar{x}, y, \bar{y})-f(x, \bar{x}, u, \bar{u})] \leq 0
$$


for all $y \in C^{m}$ and for some arbitrary sublinear functional $F$.

Let $f$ be a twice differentiable function from $C^{n} \times C^{n} \times C^{m} \times C^{m} \rightarrow C$. Let $C$ be a compact convex set in $R$, the support function of $C$ is defined by $S((x, \bar{x}) / C)=$ $\max .\left\{x^{T} y+x^{H} \bar{y},(y, \bar{y}) \in C\right\}$ for $(x, \bar{x}) \in C^{n} \times C^{n}$. A support function, being convex and everywhere finite has a sub differential in the sense of convex analysis, that is there exist $(z, \bar{z})$ such that

$$
\operatorname{Re}[S((x, \bar{x}) / C)-S((y, \bar{y}) / C)] \geq \operatorname{Re}\left[(z, \bar{z})^{T}\{(x, \bar{x})-(y, \bar{y})\}\right] \quad \text { for all }(x, \bar{x}) .
$$

The sub differential $S(x / C)$ is given by

$$
\delta S((x, \bar{x}) / C)=\left\{(z, \bar{z}) \in C^{n} \times C^{n}: z^{T} x+z^{H} \bar{x}=S((x, \bar{x}) / C)\right\} .
$$

There is a relation between normal cones and support functions of a compact convex set $C$, namely, $(y, \bar{y})$ is in $N_{C}(x, \bar{x})$ if and only if $S((y, \bar{y}) / C)=(x, \bar{x})^{T}(y, \bar{y})$.

Consider the mathematical programming problem

$$
\min f(x, \bar{x}) \text { subject to }(x, \bar{x}) \in C^{n} \times C^{n},
$$

where $f: C^{n} \times C^{n} \rightarrow C$ and $x \in C^{m} \times C^{m}$. A feasible point $\left(x_{0}, x_{0}\right)$ is said to be weak optimal solution of (1) if there exist no other feasible point $(x, \bar{x})$ for which $\operatorname{Re}\left[f\left(x_{0}, x_{0}\right)-f(x, \bar{x})\right]>0$. If a feasible point $\left(x_{0}, x_{0}\right)$ is optimal then it is clear that it is also a weak solution.

In what follws we have used the notion of generalized $F$-convexity of a particular kind namely for $F$ and $G$ which are sub linear functionals there are properties like $F$-convexity and $G$-convexity. These properties have also been used in some of the references which we have quoted in the present article. We do not repeat the details of such descriptions.

\section{Symmetric Duality}

Consider the following pair of symmetric duals of non-differentiable mathematical programs.

\section{Primal (VP)}

$$
\operatorname{Minimize} \frac{\operatorname{Re}\left[\left(f(x, \bar{x}, y, \bar{y})+S((x, \bar{x}) / C)-\left(y^{T} z+y^{H} \bar{z}\right)\right)\right]}{\operatorname{Re}\left[\left(g(x, \bar{x}, y, \bar{y})-S((x, \bar{x}) / E)+\left(y^{T} r+y^{H} \bar{r}\right)\right)\right]}=l .
$$

Subject to

(a) $\operatorname{Re}\left[\left\{\overline{\nabla_{y} f(x, \bar{x}, y, \bar{y})}-z\right\}+\left\{\nabla_{\bar{y}} f(x, \bar{x}, y, \bar{y})-\bar{z}\right\}-l\left[\left\{\overline{\nabla_{y} g(x, \bar{x}, y, \bar{y})}+r\right\}\right.\right.$

$\left.\left.+\left\{\nabla_{\bar{y}} g(x, \bar{x}, y, \bar{y})+\bar{r}\right\}\right]\right] \leq 0$,

(b) $\operatorname{Re}\left[y^{T}\left[\left\{\overline{\nabla_{y} f(x, \bar{x}, y, \bar{y})}-z\right\}-l\left\{\overline{\nabla_{y} g(x, \bar{x}, y, \bar{y})}+r\right\}\right]+y^{H}\left[\left\{\nabla_{\bar{y}} f(x, \bar{x}, y, \bar{y})-\bar{z}\right\}\right.\right.$

$\left.-l\left\{\nabla_{\bar{y}} g(x, \bar{x}, y, \bar{y})+\bar{r}\right\}\right] \geq 0$, 
(c) $(z, \bar{z}) \in D \in C^{m} \times C^{m}$ and $(r, \bar{r}) \in H \in C^{m} \times C^{m}$,

(d) $x \geq 0$.

\section{Dual (VD)}

$$
\operatorname{Minimize} \frac{\operatorname{Re}\left[f(u, \bar{u}, v, \bar{v})-S((v, \bar{v}) / D)+u^{T} w+u^{H} \bar{w}\right]}{\operatorname{Re}\left[g(u, \bar{u}, v, \bar{v})+S((v, \bar{v}) / H)-u^{T} t-u^{H} \bar{t}\right]}=L .
$$

Subject to

(e) $\operatorname{Re}\left[\left\{\overline{\left\{\nabla_{u} f(u, \bar{u}, v, \bar{v})+w\right\}}-L\left\{\overline{\left.\nabla_{u} g(u, \bar{u}, v, \bar{v})-t\right\}}\right\}+\left\{\left\{\nabla_{\bar{u}} f(u, \bar{u}, v, \bar{v})+\bar{w}\right\}\right.\right.\right.$

$-L\{\nabla \bar{u} g(u, \bar{u}, v, \bar{v})-\bar{t}\}] \geq 0$,

(f) $\operatorname{Re}\left[u^{T}\left\{\left\{\overline{\left\{\nabla_{u} f(u, \bar{u}, v, \bar{v})+w\right\}}-L\left\{\overline{\left\{\nabla_{u} g(u, \bar{u}, v, \bar{v})-t\right\}}\right\}+\left\{u^{H}\left\{\nabla_{\bar{u}} f(u, \bar{u}, v, \bar{v})+\bar{w}\right\}\right.\right.\right.\right.$

$\left.-L\left\{\nabla_{\bar{u}} g(u, \bar{u}, v, \bar{v})-\bar{t}\right\}\right] \leq 0$,

(g) $(w, \bar{w}) \in C \in C^{n} \times C^{n}$ and $(t, \bar{t}) \in E \in C^{n} \times C^{n}$,

(h) $v \geq 0$.

$C$ and $E$ are compact convex sets in $C^{n} \times C^{m}$ and $D$ and $H$ are compact convex sets in $C^{m} \times C^{m}$. Now we established weak and strong duality theorem between (VP) and (VD).

Theorem 1.1 (Weak Duality Theorem). Let $(x, \bar{x}, y, \bar{y}, z, \bar{z}, r, \bar{r})$ be feasible for (VP) and let $(u, \bar{u}, v, \bar{v}, w, \bar{w}, t, \bar{t})$ be feasible for (VD).

$$
\begin{gathered}
\text { Let } \operatorname{Re}\left[\left\{f(., ., v, \bar{v})+(.)^{T} w+(.)^{H} \bar{w}\right\}-L\left\{g(., ., v, \bar{v})-(.)^{T} t-(.)^{H} \bar{t}\right\}\right] \\
\text { be F-Pseudoconvex at }(u, \bar{u}) . \\
\text { And let } \operatorname{Re}\left[\left\{f(x, \bar{x}, ., .)-(.)^{T} z-(.)^{H} \bar{z}\right\}-l\left\{f(x, \bar{x}, . . .)+(.)^{T} r+(.)^{H} \bar{r}\right\}\right] \\
\text { be G-Pseudoconvex at }(y, \bar{y}) .
\end{gathered}
$$

Assume that,

$$
\begin{aligned}
& F\left(x, u,\left\{\overline{\nabla_{u} f(u, \bar{u}, v, \bar{v})+w}+\nabla_{\bar{u}} f(u, \bar{u}, v, \bar{v})+\bar{w}\right\}\right. \\
& \left.\left.-L\left\{\overline{\nabla_{u} g(u, \bar{u}, v, \bar{v})-t}+\nabla_{\bar{u}} f(u, \bar{u}, v, \bar{v})-\bar{t}\right\}\right\}\right) \\
& +\operatorname{Re}\left[u^{T}\left\{\overline{\left(\nabla_{u} f(u, \bar{u}, v, \bar{v})+w\right)}-L \overline{\left(\nabla_{u} g(u, \bar{u}, v, \bar{v})-t\right)}\right\}\right. \\
& \left.+u^{H}\left\{\left(\nabla_{\bar{u}} f(u, \bar{u}, v, \bar{v})+\bar{w}\right)-L\left(\nabla_{\bar{u}} g(u, \bar{u}, v, \bar{v})-\bar{t}\right)\right\}\right] \geq 0 \\
& \quad G\left(v, y,\left\{\overline{\nabla_{y} f(x, \bar{x}, y, \bar{y})-z}+\nabla_{\bar{y}} f(x, \bar{x}, y, \bar{y})-\bar{z}\right\}\right. \\
& \left.\left.-l\left\{\overline{\nabla_{y} g(x, \bar{x}, y, \bar{y})+r}+\nabla_{\bar{y}} g(x, \bar{x}, y, \bar{y})+\bar{r}\right)\right\}\right) \\
& +\operatorname{Re}\left[y ^ { T } \left[\left\{\overline{\left(\nabla_{y} f(x, \bar{x}, y, \bar{y})\right.}-\bar{z}\right\}-l\left\{\overline{\nabla_{y} g(x, \bar{x}, y, \bar{y})}+\bar{r}\right]\right.\right. \\
& +y^{H}\left[\left\{\nabla_{\bar{y}} f(x, \bar{x}, y, \bar{y})+\bar{r}\right)-l\left\{\nabla_{\bar{y}} g(x, \bar{x}, y, \bar{y})+\bar{r}\right\}\right] \leq 0
\end{aligned}
$$


then

$$
\begin{aligned}
& \frac{\operatorname{Re}\left[f(x, \bar{x}, y, \bar{y})+S((x, \bar{x}) / C)-y^{T} z-y^{H} \bar{z}\right]}{\operatorname{Re}\left[g(x, \bar{x}, y, \bar{y})-S((x, \bar{x}) / E)+y^{T} r+y^{H} \bar{r}\right]} \\
\geq & \frac{\operatorname{Re}\left[f(u, \bar{u}, v, \bar{v})-S((v, \bar{v}) / D)+u^{T} w+u^{H} \bar{w}\right]}{\operatorname{Re}\left[g(u, \bar{u}, v, \bar{v})+S((v, \bar{v}) / H)-u^{T} t-u^{H} \bar{t}\right]}
\end{aligned}
$$

Here it is assumed that the numerator of the objective function is nonnegative and the denominator is positive in the feasible region for both the primal and dual problems.

Proof. From (4)

$$
\begin{aligned}
& F\left(x, u,\left\{\overline{\nabla_{u} f(u, \bar{u}, v, \bar{v})+w}+\nabla_{\bar{u}} f(u, \bar{u}, v, \bar{v})+\bar{w}\right\}\right. \\
& \left.\left.-L\left\{\overline{\nabla_{u} g(u, \bar{u}, v, \bar{v})-t}+\nabla_{\bar{u}} f(u, \bar{u}, v, \bar{v})-\bar{t}\right\}\right\}\right) \\
& +\operatorname{Re}\left[u^{T}\left\{\overline{\left(\nabla_{u} f(u, \bar{u}, v, \bar{v})+w\right)}-L \overline{\left(\nabla_{u} g(u, \bar{u}, v, \bar{v})-t\right)}\right\}\right. \\
& \left.+u^{H}\left\{\left(\nabla_{\bar{u}} f(u, \bar{u}, v, \bar{v})+\bar{w}\right)-L\left(\nabla_{\bar{u}} g(u, \bar{u}, v, \bar{v})-\bar{t}\right)\right\}\right] \geq 0
\end{aligned}
$$

since $\operatorname{Re}\left[\left[f(., ., v, \bar{v})+(.)^{T} w+(.)^{H} \bar{w}\right]-L\left[g(., ., v, \bar{v})-(.)^{T} t-(.)^{H} \bar{t}\right]\right]$ is $F$-Pseudoconvex at $(u, \bar{u})$, it follows that

$$
\begin{aligned}
& \left.\operatorname{Re}\left[f(x, \bar{x}, v, \bar{v})+(x)^{T} w+(x)^{H} \bar{w}\right]-L\left[g(x, \bar{x}, v, \bar{v})+S((v, \bar{v}) / H)-(x)^{T} t-(x)^{H} \bar{t}\right]\right] \\
& \left.-\operatorname{Re}\left[f(u, \bar{u}, v, \bar{v})+(u)^{T} w+(u)^{H} \bar{w}\right]-L\left[g(u, \bar{u}, v, \bar{v})+S((v, \bar{v}) / H)-(u)^{T} t-(u)^{H} \bar{t}\right]\right] \geq 0
\end{aligned}
$$

I.e. $\operatorname{Re}\left[f(x, \bar{x}, v, \bar{v})-S((v, \bar{v}) / D)+(x)^{T} w+(x)^{H} \bar{w}\right]-L\left[g(x, \bar{x}, v, \bar{v})+S((v, \bar{v}) / H)-(x)^{T} t-\right.$ $\left.\left.(x)^{H} \bar{t}\right]\right] \geq 0$

$\operatorname{Re}\left[(x)^{T} w+(x)^{H} \bar{w}\right] \leq \operatorname{Re}[S((x, \bar{x}) / C)]$ and from (7), then from

$\operatorname{Re}\left[(x)^{T} t+(x)^{H} \bar{t}\right] \leq \operatorname{Re}[S((x, \bar{x}) / E)],(t, \bar{t}) \in E$,

$\operatorname{Re}\left[(v)^{T} r+(v)^{H} \bar{r}\right] \leq \operatorname{Re}[S((v, \bar{v}) / H)],(r, \bar{r}) \in H$,

$\operatorname{Re}\left[f(u, \bar{u}, v, \bar{v})+S((v, \bar{v}) / H)-u^{T} t-u^{H} \bar{t}\right]>0$

by (7), we obtain

$$
\begin{aligned}
& \operatorname{Re}[f(x, \bar{x}, v, \bar{v})+S((x, \bar{x}) / C)-S((v, \bar{v}) / D)] \\
& \left.-L\left[g(x, \bar{x}, v, \bar{v})-S((x, \bar{x}) / E)+(v)^{T} r+(v)^{H} \bar{r}\right]\right] \geq 0
\end{aligned}
$$

From (5)

$$
\begin{aligned}
& G\left(v, y,\left\{\overline{\nabla_{y} f(x, \bar{x}, y, \bar{y})-z}+\nabla_{\bar{y}} f(x, \bar{x}, y, \bar{y})-\bar{z}\right\}\right. \\
& \left.-l\left\{\overline{\nabla_{y} g(x, \bar{x}, y, \bar{y})+r}+\nabla_{\bar{y}} g(x, \bar{x}, y, \bar{y})+\bar{r}\right)\right\} \\
& +\operatorname{Re}\left[y^{T}\left[\left\{\overline{\left(\nabla_{y} f(x, \bar{x}, y, \bar{y})-z\right.}-\overline{\left(\nabla_{y} g(x, \bar{x}, y, \bar{y})+r\right.}\right\}\right]\right. \\
& \left.\left.+y^{H}\left[\left\{\nabla_{\bar{y}} f(x, \bar{x}, y, \bar{y})-\bar{z}\right)-\nabla_{\bar{y}} g(x, \bar{x}, y, \bar{y})+\bar{r}\right\}\right]\right] \leq 0
\end{aligned}
$$


From $\operatorname{Re}\left[\left\{f(x, \bar{x}, . .)-.(.)^{T} z-(.)^{H} \bar{z}\right\}-l\left\{g(x, \bar{x}, . .)-.S((x, \bar{x}) / E)+(.)^{T} r+(.)^{H} \bar{r}\right\}\right] G-$ pseudoconcave at $(y, \bar{y})$, it implies that,

$$
\begin{aligned}
& \operatorname{Re}\left[\left\{f(x, \bar{x}, v, \bar{v})-(v)^{T} z-(v)^{H} \bar{z}\right\}\right. \\
& \left.-l\left\{g(x, \bar{x}, v, \bar{v})-S((x, \bar{x}) / E)+(v)^{T} r+(v)^{H} \bar{r}\right\}\right] \\
& -\operatorname{Re}\left[\left\{f(x, \bar{x}, y, \bar{y})-(y)^{T} z-(y)^{H} \bar{z}\right\}\right. \\
& \left.-l\left\{g(x, \bar{x}, y, \bar{y})-S((x, \bar{x}) / E)+(y)^{T} r+(y)^{H} \bar{r}\right\}\right] \leq 0 \\
& \operatorname{Re}\left[\left\{f(x, \bar{x}, v, \bar{v})+S((x, \bar{x}) / C)-(v)^{T} z-(v)^{H} \bar{z}\right\}\right. \\
& \left.-l\left\{g(x, \bar{x}, v, \bar{v})-S((x, \bar{x}) / E)+(v)^{T} r+(v)^{H} \bar{r}\right\}\right] \leq 0
\end{aligned}
$$

since $\operatorname{Re}\left[v^{T} z+v^{H} \bar{z}\right] \leq \operatorname{Re}[S((v, \bar{v}) / D)](z, \bar{z}) \in D \in C^{m} \times C^{m}$.

$\operatorname{Re}\left[(x)^{T} t+(x)^{H} \bar{t}\right] \leq \operatorname{Re}\left[S((t, \bar{t}) / E],(t, \bar{t}) \in E \in C^{m} \times C^{m}\right.$

$\operatorname{Re}\left[(v)^{T} r+(v)^{H} \bar{r}\right] \leq \operatorname{Re}[S((v, \bar{v}) / H],(r, \bar{r}) \in H$, and because

$\operatorname{Re}\left[f(x, \bar{x}, y, \bar{y})+S((x, \bar{x}) / C)-y^{T} z-y^{H} \bar{z}\right] \geq 0$,

$\operatorname{Re}\left[g(x, \bar{x}, y, \bar{y})-S((x, \bar{x}) / E)+y^{T} r+y^{H} \bar{r}\right]>0$, we get

$$
\begin{aligned}
& \operatorname{Re}[\{f(x, \bar{x}, v, \bar{v})+S((x, \bar{x}) / C)-S((v, \bar{v}) / D)\} \\
& \left.-l\left\{g(x, \bar{x}, v, \bar{v})-S((x, \bar{x}) / E)+(v)^{T} r+(v)^{H} \bar{r}\right\}\right] \leq 0
\end{aligned}
$$

Now, Combining (9) and (10), we have

$$
(l-L)\left\{g(x, \bar{g}, v, \bar{v})-S((x, \bar{x}) / E)+(v)^{T} r+(v)^{H} \bar{r}\right\} \geq 0
$$

Since $\operatorname{Re}\left[g(x, \bar{x}, y, \bar{y})-S((x, \bar{x}) / E)+y^{T} r+y^{H} \bar{r}\right]>0,(11)$ implies $(l) \geq(L)$

Theorem 1.2 (Strong Duality Theorem). If $\left(x^{0}, \bar{x}^{0}, y^{0}, \bar{y}^{0}, z^{0}, \bar{z}^{0}\right)$ is an optimal solution for (VP) then there exist $\left(w^{0}, \bar{w}^{0}\right) \in C^{n} \times C^{n}$, such that $\left(x^{0}, \bar{x}^{0}, y^{0}, \bar{y}^{0}, w^{0}, \bar{w}^{0}\right)$ is feasible for (VD) and let the assumption of Theorem 1.1 be fulfilled. Assume that,

(i) $\operatorname{Re}\left[\overline{\left(\nabla_{y y}+\nabla_{y \bar{y}}\right) f}+\left(\nabla_{\bar{y} y}+\nabla_{\overline{y y}}\right) f\right]$ is + ive or - ive definite. then $\left(x^{0}, \bar{x}^{0}, y^{0}, \bar{y}^{0}, w^{0}, \bar{w}^{0}\right)$ is a solution for (VD) and both (VP) and (VD) have the same optimal value.

Proof. A strong duality theorem for this type of problems can be proved in the single objective view of $[6]$ in the light of the above theorem.

\section{Conclusion and Special Cases.}

It has been shown in many of the eariler works ([5] and [6]) non smooth programming duality can be tackled by introducing quadratic terms like $\left(x^{T} A x\right)^{1 / 2}$. Even in the fractional objective case the numerator as well as denominator can contain such quadratic terms. 


\section{Special cases:}

If we are taking real case: A frequently occuring example of a non differentiable support function is $\left(x^{T} A x\right)^{1 / 2}$, where $A$ is a positive semi definite matrix. It can be easily verified that $\left(x^{T} A x\right)^{1 / 2}=S(x / C)$, where $C=\left\{A y:\left(y^{T} A y\right) \leq 1\right\}$ and that set $C$ is compact convex.

(a) If in the feasible regions $k \equiv 1, g_{i} \equiv 1,\left(x_{i}^{T} B x\right)^{1 / 2}=S\left(x / C_{i}\right)$, where $C_{i}=\left\{B_{i} y\right.$ : $\left.\left(y^{T} B_{i} y\right) \leq 1\right\},\left(x^{T} C x\right)^{1 / 2}=S\left(x / D_{i}\right)$, where

$$
D_{i}=\left\{C_{i} y:\left(y^{T} C_{i} y\right) \leq 1\right\}, \quad i=1,2, \ldots, k,
$$

then programs (FP) and (FD) become a pair of symmetric dual non differentiable programs considered by Chandra, Craven, and Mond [2].

(b) If in (FP) and (FD), $B_{i}=\{0\}$ and $C_{i}=\{0\}, i=1,2, \ldots, k$, and in the feasible regions $g_{i} \equiv 1, i=1,2, \ldots, k$, we obtain the symmetric dual multiobjective programming problems studied by Weir and Mond [4].

(c) If in (FP) and (FD), $k \equiv 1, g_{i} \equiv 1, C_{i}=\{0\}$ and $D_{i}=\{0\}, i=1,2, \ldots, k$, we obtain symmetric dual problems studied by Chandra, Craven, and Mond [2].

(d) If in (FP) and (FD), $k \equiv 1, g_{i} \equiv 1$, we obtain symmetric dual problems studied by Mond and Schecher [1].

(e) If we consider the real case, then it reduces in the single objective view of [6].

(f) If we consider the Integral real case, then it reduces in the single objective view of [5].

2. If we are eliminating support functions then it will reduce to work in [3] for $1^{\text {st }}$ order case.

\section{References}

[1] B. Mond and M. Schechter, Nondifferentiable symmetric duality, bull. Austral. Math. Soc. 53(1996), 177-188.

[2] S. Chandra, B. D. Craven and B. Mond, Symmetric dual fractional programming, Z. Oper. Res. 29(1985), 59-64.

[3] S. K. Mishra and Norma G. Rueda, Symmetric duality for mathematical programming in complex spaces with F-convexity, J. Math. Anal. Appl. 284(2003), 250-265.

[4] T. Weir and B. Mond, Generalized convexity and duality for complex programming problem, Cahiers Centre Etudes Rech. Oper. 26(1984), 137-142.

[5] Xin min Yang, S. Wang and Duan Li, Symmetric duality for a class of multi objective programming, International J. of Math. Sciences 24(2000), 617-625.

[6] Xin min Yang, S. Y. Wang and X. T. Deng, Symmetric duality for a class of multiobjective fractional programming problems, J. Math. Anal. Appl. 274(2002), 279-295.

Department of Applied Mathematics, Institute of Technonlogy Banaras Hindu University, Varanasi, India (221005).

E-mail: ojhdb@yahoo.co.in 